Origin of the Pseudogap in High Temperature Superconductors

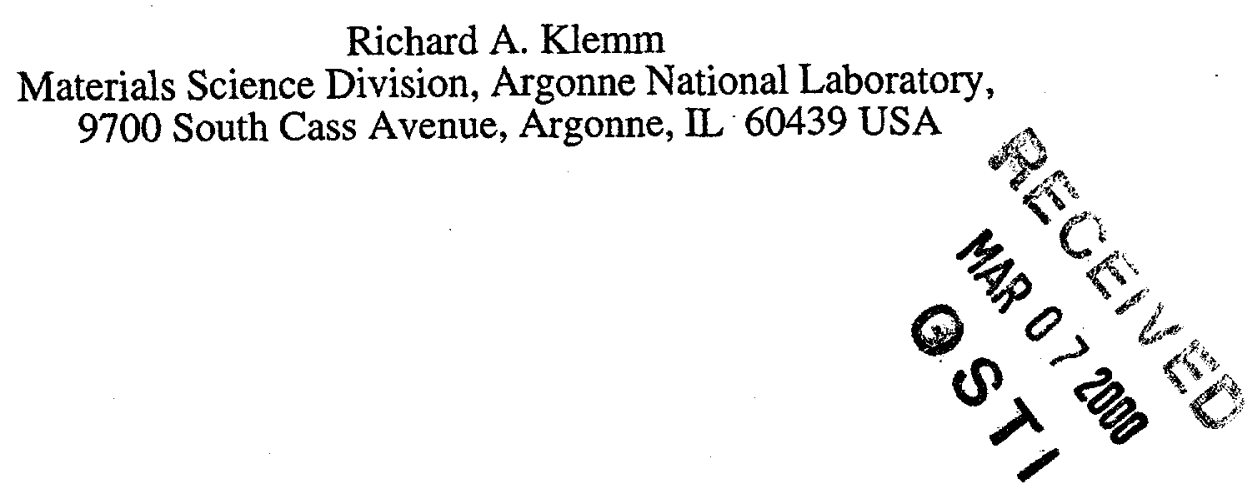

Invited talk at the $6^{\text {th }}$ International Conference on Materials and Mechanisms of Superconductivity and High Temperature Superconductors, Houston, TX, February 18-25, 2000. PHYSICA C

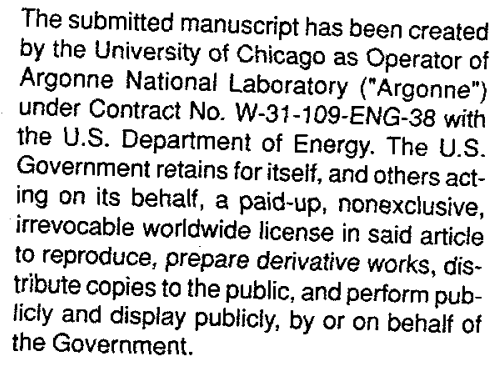

This work was supported by the U.S. Department of Energy, Office of Basic Energy Sciences Division of Materials Sciences, under contract \#W-31-109-ENG-38. 


\section{DISCLAIMER}

This report was prepared as an account of work sponsored by an agency of the United States Government. Neither the United States Government nor any agency thereof, nor any of their employees, make any warranty, express or implied, or assumes any legal liability or responsibility for the accuracy, completeness, or usefulness of any information, apparatus, product, or process disclosed, or represents that its use would not infringe privately owned rights. Reference herein to any specific commercial product, process, or service by trade name, trademark, manufacturer, or otherwise does not necessarily constitute or imply its endorsement, recommendation, or favoring by the United States Government or any agency thereof. The views and opinions of authors expressed herein do not necessarily state or reflect those of the United States Government or any agency thereof. 


\section{DISCLAIMER}

Portions of this document may be illegible in electronic image products. Images are produced from the best available original document. 


\title{
Origin of the Pseudogap in High Temperature Superconductors
}

\author{
R. A. Klemm ${ }^{\mathrm{a} *}$ \\ ${ }^{a}$ Materials Science Division, Argonne National Laboratory, Argonne, IL 60439 USA
}

Underdoped high temperature superconductors (HTS) exhibit a 'normal' state for energies $E>E_{g}$ and/or temperatures $T>T_{0}$, and a pseudogap in their electronic spectrum for $E<E_{g}$ and/or $T_{0}>T>T_{c}$. Strikingly similar behavior occurs in the transition metal dichalcogenides (TMD) $2 H-\mathrm{MX}_{2}$, where $\mathrm{M}=\mathrm{Ta}, \mathrm{Nb}$, and $\mathrm{X}=$ $\mathrm{S}$, Se, both in the 'normal' $\left(T>T_{0}\right)$ and in the incommensurate charge-density wave $\left(T_{I C D W}>T>T_{c}\right)$ states. Such strikingly similar behavior has also been seen in the organic layered superconductors (OLS) $\kappa$ - $(\mathrm{ET})_{2} \mathrm{X}$, where ET is bis(ethylenedithio)tetrathiafulvalene, and $\mathrm{X}=\mathrm{Cu}\left[\mathrm{N}(\mathrm{CN})_{2}\right] \mathrm{Cl}, \mathrm{Cu}\left[\mathrm{N}(\mathrm{CN})_{2}\right] \mathrm{Br}$, and $\mathrm{Cu}(\mathrm{SCN})_{2}$, both in the 'normal' region $T>T_{S D W}>T_{c}$ and in the spin-density wave region $T_{S D W}>T>T_{c}$. In all three materials classes, the anomalous transport and thermodynamic properties associated with the pseudogap or density-wave regime are completely independent of the applied magnetic field strength, whereas the same properties below $T_{c}$ are all strongly field-dependent. Hence, we propose that the pseudogap in the HTS arises from charge- and/or spin-density waves, and not from either superconducting fluctuations or "preformed" charged quasiparticle pairs.

The mechanism for superconductivity in the high temperature superconductors (HTS) is a continuing topic of great debate. Most workers consider the important keys to unlocking the secrets of the mechanism for superconductivity to be found either by determining the symmetry of the superconducting order parameter (OP), or by understanding the nature of the 'normal' state (NS). Although many experiments have been interpreted as giving evidence for an OP of $d_{x^{2}-y^{2}-}$ wave symmetry in the HTS, [1] some very recent experiments supported an OP with a dominant component of $s$-wave symmetry, and some were completely inconsistent with an OP of $d_{x^{2}-y^{2-}}$ wave symmetry. $[2,3]$ Thus, there is no consensus on the symmetry of the OP in the HTS.

This NS is denoted 'normal' only in the sense that it appears in the high temperature $T$ regime, but the transport and thermodynamic behavior of the NS are rather different from what is usually found above the superconducting transition temperature $T_{c}$ in conventional superconductors. In addition, depending upon the hole doping concentration $x$, there is a $T$ regime over which a pseudogap appears in the electronic spec-

\footnotetext{
-Supported by the USDOE-BES through Contract No.
} W-31-109-ENG-38. trum of the quasiparticles. This pseudogap is evidenced not only in angle-resolved photoemission spectroscopy (ARPES) experiments, [4] but also in optical reflectivity, [5], nuclear magnetic resonance (NMR), [6,7], transport, [8-10], Knight shift, [8] thermodynamic measurements, $[11,12]$ and neutron scattering experiments. $[13,14]$ In most experiments, the pseudogap features become prominent below one or two cross-over 'pseudogap temperatures' $T_{0}$ and $T^{*}$, where generally $T_{0}>T^{*}>T_{c}$. In others, [7] the pseudogap was sometimes characterized by a "pseudogap energy' $E_{g}$, unrelated to the maximum value $2|\Delta|$ of the superconducting energy gap. A qualitative $T$ versus $x$ phase diagram of the former characterization of the HTS is shown in Fig. 1. In this diagram, the region of the pseudogap is largest in the 'underdoped' part of the phase diagram [to the left of the maximum in $\left.T_{c}(x)\right]$. It is a matter of considerable ongoing debate as to whether or not this pseudogap is a precursor of the actual gap in the electronic spectrum in the superconducting state below $T_{\mathrm{c}}$. Here we argue, based upon considerable experimental evidence, that the pseudogap has a qualitatively different origin than does the superconducting gap.

Recently, two groups presented scanning tun- 


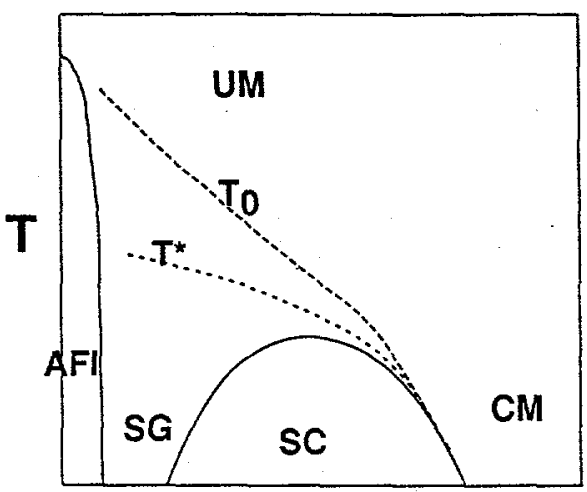

Doping $x$

Figure 1. Sketch of the $T$ vs. $x$ phase diagram of HTS. The antiferromagnetic insulator (AFI), spin-glass (SG), superconducting (SC), conventional metal (CM) and unconventional metal (UM) regions are indicated.

neling microscopy (STM) density of states data for what was claimed to be overdoped $\mathrm{Bi}_{2} \mathrm{Sr}_{2} \mathrm{CaCu}_{2} \mathrm{O}_{8+\delta}$. $[15,16]$ Both data showed a pseudogap well above $T_{c}$, which is somewhat inconsistent with Fig. 1. Regardless of the stoichiometry, careful analysis of the pseudogap peak to peak width showed that the data were most easily described in terms of two gaps. $[15,16]$ The overall behavior fit two BCS gap functions, one with an onset of $T_{0}>T_{c}$, and one with an onset $T_{o}$ satisfying $T_{0}>T_{0}>T_{c}$. Thus, these tunneling data strongly suggest that the origins of the pseudogap and of the superconducting gap are distinctly different.

Our main point is that both the behaviors in the NS and the pseudogap regime of the HTS [4-16] are strikingly similar to those seen in the $2 \mathrm{H}-\mathrm{MX}_{2}$ transition metal dichalcogenides (TMD), where $\mathrm{M}=\mathrm{Nb}, \mathrm{Ta}$ and $\mathrm{X}=\mathrm{S}, \mathrm{Se},[17]$ and in the $\kappa-(\mathrm{ET})_{2} \mathrm{X}$ organic layered superconductors (OLS), where ET is bis(ethylenedithio)tetrathiafulvalene, and $\mathrm{X}=$ $\mathrm{Cu}(\mathrm{SCN})_{2}, \mathrm{Cu}\left[\mathrm{N}(\mathrm{CN})_{2}\right] \mathrm{Br}$, and $\mathrm{Cu}\left[\mathrm{N}(\mathrm{CN})_{2}\right] \mathrm{Cl}$. [18] In the OLS, both these NS and 'pseudogaplike' behaviors are observed above and below their respective spin-density wave (SDW) tran-

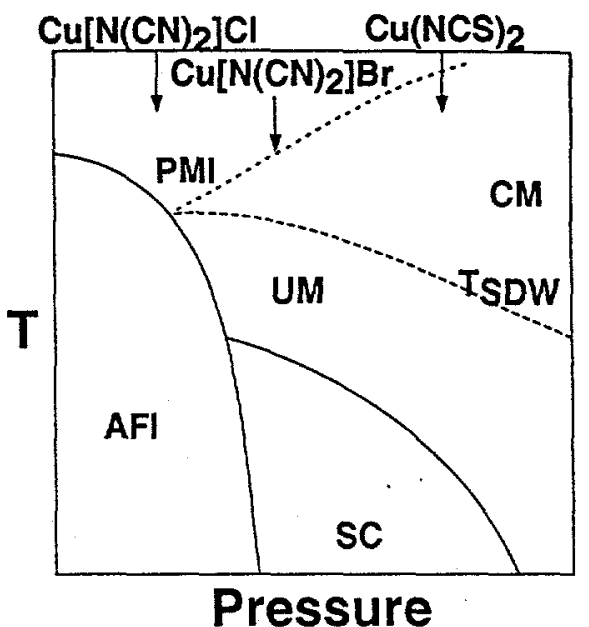

Figure 2. $T$ versus crystalline 'pressure' of the OLS $\kappa$ - $(E T)_{2} \mathrm{X}$, with $\mathrm{X}$ values indicated.. The paramagnetic insulator (PMI) region is indicated, and the other symbols are the same as in Fig. 1.

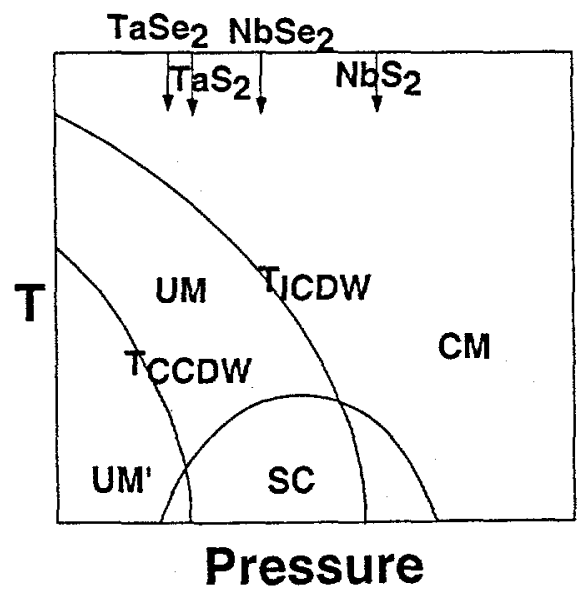

Figure 3. T versus crystalline 'pressure' of the TMD. UM and UM' represent different uncoventional metals. 
sition temperature $T_{S D W}$, indicated in Fig. 2. [19] In the TMD, these same behaviors are seen above and below their respective incommensurate charge-density wave (CDW) transition temperatures $T_{I C D W}$, indicated in Fig. 3. Although little is known from experiments about the details of the electronic structure of the OLS, both the TMD and the HTS have an NS exhibiting saddle bands, which are extended, flat regions of electronic states with saddle-like dispersions lying very near to the Fermi energy $E_{F}$, that dominate the physical behavior. The CDWs in the TMD and the pseudogap in the HTS arise from instabilities on or near to these saddle bands. [20,21] Thus, we propose that the pseudogap in the HTS arises from CDWs and/or SDWs, and not from superconducting fluctuations.

Direct evidence for static CDWs in the $2 \mathrm{H}$ $\mathrm{MX}_{2}$ TMD was very well established, using $\mathrm{X}$ ray and electron diffraction, [17] neutron diffraction, [22] and STM. [23] The second-order transitons at $T_{I C D W}$ have large $\lambda$-like specific heat anomalies (except in $2 \mathrm{H}-\mathrm{NbSe}_{2}$, where it is BCSlike), with noticeable transport and pseudogap features just below it, but the first-order transition at $T_{C C D W}$ in $2 \mathrm{H}-\mathrm{TaSe}_{2}$ exhibits a small, but hysteretic specific heat anomaly, with very subtle transport changes. [24,25] Similarly, direct evidence for long-range antiferromagnetic order in the OLS $\kappa$-(ET) ${ }_{2} \mathrm{Cu}\left[\mathrm{N}(\mathrm{CN})_{2}\right] \mathrm{Cl}$ was seen in magnetization experiments, [26] and the SDW in $\kappa$ $(\mathrm{ET})_{2} \mathrm{Cu}\left[\mathrm{N}(\mathrm{CN})_{2}\right] \mathrm{Br}$ and $\kappa$-(ET) $)_{2} \mathrm{Cu}(\mathrm{SCN})_{2}$ was seen by comparing the NMR and the magnetic susceptibility $\chi_{\perp}(T)$ for $\mathbf{H} \|$ c. [27]

Although these TMD have in-plane resistivities $\rho_{\| 1}(T)$ in the NS $T>T_{I C D W}$ that are just as linear in $T$ as are those of the HTS for $T>T_{0}$, the $\rho_{\|}(T)$ of these TMD in the CDW regime $T_{I C D W}>T>T_{c}$ are also strikingly similar to those of the underdoped HTS in the pseudogap regime below $\mathcal{T}_{0}$, becoming more 'metallic-like' there. $[8-10,25,28-30]$ In pure samples, the $c$-axis resistivity $\rho_{\perp}(T)$ of the TMD, while quasi-linear above $T_{I C D W}$ increases with decreasing $T$ below $T_{I C D W},[28]$ qualitatively similar to the behavior seen in many HTS. [10] Furthermore the values of $\rho_{\|}(300 \mathrm{~K})$ and $\rho_{\perp}(300 \mathrm{~K})$ are almost exactly the same as for the optimally doped HTS mate- rial $\mathrm{YBa}_{2} \mathrm{Cu}_{3} \mathrm{O}_{7-\delta}(\mathrm{YBCO}) .[28,29]$ The similarities of the TMD and the HTS also are present in the Hall constant $R_{H \perp}(T)$ for current $\mathbf{I} \perp \mathbf{c} \| \mathbf{H}$, and in the Seebeck coefficient. $[9,25,30]$

These similarities between the TMD and the HTS are also present in thermodynamic measurements, especially in $\chi_{\perp}(T)$, being rather $T$ independent above $T_{I C D W}$ or $T_{0}$, but decreasing with decreasing $T$ below $T_{I C D W}$ or $T_{0} .[12,17,31]$ $\chi_{\perp}(T)$ for two of the above OLS also show the same behaviors, both above and below $T_{S D W}$. [27] In addition, optical reflectivity experiments on the HTS and on one of the TMD show strikingly similar behavior. $[5,32]$ Since photons couple to the electronic charge, rather than its spin, optical reflectivity and ARPES experiments are easiest to interpret in terms of the pseudogap being a 'charge gap'. As regards the NMR and/or nuclear quadrupole resonance (NQR), the peak in $1 / T_{1} T$ in the HTS was often claimed to give evidence for a 'spin gap'.[6,7] Similar NMR behavior was indeed seen in the OLS near $T_{S D W}$. [27] In the TMD, there was only a broadening of the NMR signal at $T_{I C D W}$. [33]

One of the most interesting features of the pseudogap regime in the HTS is that all experiments that could be measured with $\mathbf{H} \neq 0$ give reulsts that are field-independent in the pseudogap regime $T_{c}<T<T_{0}$. For instance, the NMR (and NQR) $1 / \mathcal{T}_{1} T$ observed in the HTS and in the OLS are both field-independent for $T>T_{c}$, but strongly field-dependent for $T<T_{\mathrm{c}}$. $[6,27,34]$ This field-independence of the pseudogap regime combined with strong field-dependence of the superconducting state is also present in the resistivity, magnetic susceptibilty, and Hall constant in the HTS, the TMD, and the OLS, to the extent that they have been measured. $[35,30,34]$

Since superconducting fluctuations and/or 'preformed pairs' involve moving charged Boselike pair excitations, their motion always results in Landau orbits, which are diamagnetic. Thus, if the suppression in $\rho_{\|}(T)$ for $T_{c}<T<T_{0}$ were due to superconducting fluctuations, one would expect it to disappear in a strong field, but it only does so below $T_{c}$ (and in a very narrow $T$ range above $T_{c}$ ). For example, $\chi_{\perp}(T)$ in $2 H$ $\mathrm{TaS}_{2}$ (pyridine) $)_{1 / 2}$ was once reported to give ev- 
idence for measureable superconducting fluctuation effects out to $35 \mathrm{~K}$, or $10 T_{c}$. [36]. However, the presence of.CDWs in the unintercalated $2 \mathrm{H}$ $\mathrm{TaS}_{2}$ was found shortly thereafter, [17] and the observed $\chi_{\perp}(T)$ suppression was later found to be instead due to remanent CDWs present with incomplete intercalation. [31] The correct fluctuation effects were limited to a very narrow regime (up to $1.2 T_{c}$ ) above $T_{c}$, not up to $T_{I C D W}$.[37]

Since the properties of the pseudogap regime in the HTS are very similar to those of the CDW regime in the TMD and of the SDW regime in the OLS, they are independent of the applied magnetic field strength, and STM measurements indicate two distinct 'gaps', we propose that the pseudogap in the HTS is not due to superconducting fluctuations (or 'preformed pairs'), but instead due to CDW and/or SDW formation.

\section{REFERENCES}

1. C. C. Tsuei et al., Phys. Rev. Lett. 73, 593 (1994).

2. Q. Li et al., Phys. Rev. Lett. 83, 4160 (1999).

3. A. Bhattacharya et al., Phys. Rev. Lett. 82, 3132 (1999); M. Mößle and R. Kleiner, Phys. Rev. B 59, 4486 (1999).

4. H. Ding et al., Nature 382, 51 (1996).

5. D. N. Basov et al., Science 283, 49 (1999).

6. G.-q. Zheng et al., Phys. Rev. B 60, R9947 (1999); K. Gorny et al., Phys. Rev. Lett. 82, 177 (1999).

7. G. V. Williams et al., Phys. Rev. Lett. 78, 721 (1997).

8. B. Wuyts, V. V. Moschalkov, and Y. Bruynseraede, Phys. Rev. B 53, 9418 (1996).

9. T. Tamegai and Y. Iye, Phys. Rev. B 44, 10 167 (1991).

10. T. Watanabe, T. Fujii, and A. Matsuda, Phys. Rev. Lett. 79, 2113 (1997).

11. J. W. Loram et al., Phys. Rev. Lett. 71, 1740 (1993).

12. D. C. Johnston, Phys. Rev. Lett. 60, 762 (1988).

13. H. F. Fong et al., Phys. Rev. Lett. 82, 1939 (1999).

14. P. Dai, H. A. Mook, and F. Dogan, Phys. Rev. Lett. 80, 1738 (1998).
15. T. Ekino, Y. Sezaki, and H. Fujii, Phys. Rev. B 60, 6916 (1999).

16. Ch. Renner et al., Phys. Rev. Lett. 80, 149 (1998); M. Franz and A. J. Millis, Phys. Rev. B 58, 14572 (1998).

17. J. A. Wilson, F. J. DiSalvo, and S. Mahajan, Adv. Phys. 24, 117 (1974).

18. J. M. Williams et al., Organic Superconductors (Including Fullerenes) (Prentice-Hall, Englewood Cliffs, NJ, 1992).

19. R. McKenzie, Science 278, 820 (1997).

20. R. Liu et al., Phys. Rev. Lett. 80, 5762 (1998).

21. D. S. Marshall et al., Phys. Rev. B 52, 12548 (1995).

22. D. E. Moncton, J. D. Axe, and F. J. DiSalvo, Phys. Rev. B 16, 801 (1977).

23. R. V. Coleman et al., Adv. Phys. 37, 559 (1988).

24. R. A. Craven and S. R. Meyer, Phys. Rev. B 16,4583 (1977).

25. M. Naito and S. Tanaka, J. Phys. Soc. Jpn. 51, 219 (1982).

26. K. Miyagawa et al., Phys. Rev. Lett. 75, 1174 (1995).

27. A. Kawamoto et al., Phys. Rev. Lett. 74, 3455 (1995).

28. J. P. Tidman et al., Phil. Mag. 30, 1191 (1974).

29. T. A. Friedmann et al., Phys. Rev. B 42,6217 (1990).

30. D. J. Huntley and R. F. Frindt, Can. J. Phys. 52, 861 (1974).

31. F. J. DiSalvo, in Low Temperature PhysicsLT13, K. D. Timmerhaus et al., Eds. (Plenum, New York, 1974) 3, pp. 417-427.

32. V. Vescoli et al., Phys. Rev. Lett. 81, 453 (1998).

33. K. Ishida et al., J. Phys. Soc. Jpn. 65, 2341 (1996).

34. H. Mayaffre et al., Phys. Rev. Lett. 75, 4122 (1995).

35. W. Kwok, private communication.

36. T. H. Geballe et al., Phys. Rev. Lett. 27, 314 (1971).

37. D. E. Prober, M. R. Beasley, and R. E. Schwall, Phys. Rev. B 15, 5245 (1977). 